\title{
What is the blood concentration of extracellular vesicles? Implications for the use of extracellular vesicles as blood-borne biomarkers of cancer
}

Johnsen, Kasper Bendix; Gudbergsson, Johann Mar; Andresen, Thomas Lars; Simonsen, Jens Bæk

Published in:

BBA Reviews on Cancer

Link to article, DOI:

10.1016/j.bbcan.2018.11.006

Publication date:

2019

Document Version

Peer reviewed version

Link back to DTU Orbit

Citation (APA):

Johnsen, K. B., Gudbergsson, J. M., Andresen, T. L., \& Simonsen, J. B. (2019). What is the blood concentration of extracellular vesicles? Implications for the use of extracellular vesicles as blood-borne biomarkers of cancer. BBA Reviews on Cancer, 1871(1), 109-116. https://doi.org/10.1016/j.bbcan.2018.11.006

\section{General rights}

Copyright and moral rights for the publications made accessible in the public portal are retained by the authors and/or other copyright owners and it is a condition of accessing publications that users recognise and abide by the legal requirements associated with these rights.

- Users may download and print one copy of any publication from the public portal for the purpose of private study or research.

- You may not further distribute the material or use it for any profit-making activity or commercial gain

- You may freely distribute the URL identifying the publication in the public portal 


\section{Accepted Manuscript}

What is the blood concentration of extracellular vesicles? Implications for the use of extracellular vesicles as blood-borne biomarkers of cancer

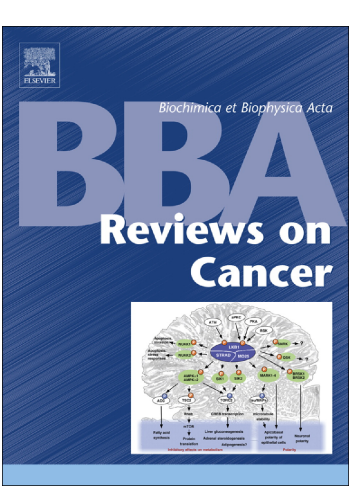

Kasper Bendix Johnsen, Johann Mar Gudbergsson, Thomas Lars Andresen, Jens Bæk Simonsen

PII: $\quad$ S0304-419X(18)30180-X

DOI: $\quad$ https://doi.org/10.1016/j.bbcan.2018.11.006

Reference: $\quad$ BBACAN 88259

To appear in: $\quad B B A-$ Reviews on Cancer

Received date: $\quad 29$ October 2018

Revised date: $\quad 16$ November 2018

Accepted date: $\quad 20$ November 2018

Please cite this article as: Kasper Bendix Johnsen, Johann Mar Gudbergsson, Thomas Lars Andresen, Jens Bæk Simonsen, What is the blood concentration of extracellular vesicles? Implications for the use of extracellular vesicles as blood-borne biomarkers of cancer. Bbacan (2018), https://doi.org/10.1016/j.bbcan.2018.11.006

This is a PDF file of an unedited manuscript that has been accepted for publication. As a service to our customers we are providing this early version of the manuscript. The manuscript will undergo copyediting, typesetting, and review of the resulting proof before it is published in its final form. Please note that during the production process errors may be discovered which could affect the content, and all legal disclaimers that apply to the journal pertain. 
What is the blood concentration of extracellular vesicles? Implications for the use of extracellular vesicles as blood-borne biomarkers of cancer

Kasper Bendix Johnsen ${ }^{1 *}$ kasjoh@nanotech.dtu.dk, Johann Mar Gudbergsson ${ }^{2}$, Thomas Lars Andresen ${ }^{1} \&$ Jens Bæk Simonsen ${ }^{1 *}$ jbak@ nanotech.dtu.dk

${ }^{1}$ Center for Nanomedicine and Theranostics, Department of Micro- and Nanotechnology, Technical University of Denmark, Denmark.

${ }^{2}$ Laboratory of Immunology and Cancer Biology, Department of Health Science and Technology, Aalborg University, Denmark.

*Corresponding authors at: Center for Nanomedicine and Theranostics, Department of Micro- and Nanotechnology, Technical University of Denmark, Produktionstorvet, Building 423, Room 212 2800 Kongens Lyngby, Denmark.

\begin{abstract}
Circulating biomarkers have a great potential in diagnosing cancer diseases at early stages, where curative treatment is a realistic possibility. In the recent years, using extracellular vesicles (EVs) derived from blood as biomarkers has gained widespread popularity, mainly because they are thought to be easy to isolate and carry a vast variety of biological cargos that can be analyzed for biomarker purposes. However, our current knowledge on the plasma EV concentration in normophysiological states is sparse. Here, we provide the very first mean estimate of the plasma EV concentration based on values obtained from a thorough literature review. The different estimates obtained from the literature are correlated to the isolation techniques used to obtain them, illustrating how some methodologies may over- or underestimate the plasma EV concentration. We also show that the estimated plasma EV concentration (approximately $10^{10} \mathrm{EVs}$ per $\mathrm{mL}$ ) defines EVs as a minority population compared to other colloidal particles of the systemic circulation, namely the lipoproteins, which are known contaminants in EV isolates and carry biomarker molecules themselves. Lastly, we introduce the possibility of regarding EVs and lipoproteins as a continuum of lipid-containing particles to which biomarker molecules can be associated. Using such a holistic approach, increased strength of plasma-derived cancer biomarkers may soon be revealed.
\end{abstract}

\title{
Introduction
}

Extracellular vesicles (EVs) have gained wide popularity within the past decade due to their role in intercellular communication and disease biology, and their potential in biomarker development and drug delivery [1-3]. The EVs are lipid bilayer particles composed of a range of different lipids and proteins (especially phospholipids, cholesterol and tetraspanin proteins), whereas they can carry proteins, RNA and DNA in their aqueous core (Figure 1A)[3]. They are produced by most cells of the body, also under pathological conditions such as cancer [4]. The EVs secreted by cancer cells can be used as a signature of disease progression, severity, and therapeutic efficacy [2]. In the recent years, there has been a strong focus on the use of EVs as such biomarkers of disease (Figure 1B)[5-7]. The progression of the research field is driven by collaborative efforts between clinicians with access to patients, and basic researchers with the analytical expertise to study the biological material obtained from the patients [6]. Due to easy access to blood sampling and the extent of the general information possessed in such samples, this type of biological material has been the choice for most of the biomarker studies focusing on EVs. From the original sample, EVs are isolated either from plasma or serum using standard isolation methods available in the EV community [4]. Published work in the recent years has highlighted that it is very difficult to obtain a pure sample of EVs from blood plasma or serum because several types of lipoproteins contaminate the EV samples [8-11]. Lipoproteins are biological particles responsible for the transportation of lipids throughout the body. The lipoprotein family is comprised of five subclasses including chylomicrons, very-low-density 
lipoproteins (VLDL), intermediate density lipoproteins (IDL), low-density lipoproteins (LDL), and highdensity lipoproteins (HDL).

Here we discuss the reported EV levels in human blood plasma based on a literature survey and the proposed lipoprotein contamination as a function of the methods used to isolate and detect the EVs. The key problem discussed is the important but highly ignored fact that EVs are a minority population compared to other colloidal particles in blood (lipoproteins) - a fact which was recently called for information on [3]. The fact that EVs are also a minority population to red blood cells and platelets is not considered here [8]. We show based on this discussion that there are 6 orders of magnitude higher total lipoprotein particle concentration compared to the EV concentration in plasma. The combination of the physical properties of lipoproteins and EVs [9] and the much higher level of lipoproteins compared to EVs drives the lipoprotein contamination. Therefore, we stress the importance of considering the lipoprotein contamination when interpreting biologically relevant functions of EVs.

\section{What is the EV concentration in human blood?}

EVs are enumerated using various analytical procedures to obtain a concentration that can be correlated to the concentration of different biomarker molecules, e.g. DNAs, RNAs, proteins, and lipids, contained within the EVs (Figure 1A)[3]. Enumeration of EVs can also in itself be used as a biomarker for different cancers, since these diseases are characterized by a remarkable increase in total plasma levels of the EVs [7,10-15]. This is hypothesized to be mediated by $\mathrm{pH}$ reduction in the tumor microenvironment, which stimulates EV production [7,12-16]. Despite the large popularity of enumerating EVs in blood for different purposes, there is still no accepted baseline value that could be used as a setpoint for other studies. The reason for this is likely multifaceted but does include the differences in both isolation and quantification methodologies [17], and lack of reproducibility of findings [8]. This makes comparisons between different studies difficult.

We argue here that a baseline concentration of EVs in blood from healthy humans can be estimated, if data from many studies across different isolation and quantification methodologies are obtained. Through a literature survey including studies from 2013 - 2018 (the years of rapid growth in the publication frequency and of significant improvement in isolation and quantification methodologies, Figure 1B), we identified 38 original articles that provided 59 estimates of the EV concentration in blood from healthy human subjects $[10,13,18-53]$. Only studies that provided a particle concentration were included, whereas studies providing only EV protein concentration with no correlation to the absolute numbers of EVs were left out. EV concentrations in subjects with disease or obtained from other species were also not considered. Thematic analysis of all the studies published between 2013 and 2018 revealed that the vast majority of published studies used EVs as biomarkers in cancer, whereas disease groups like cardiovascular, infectious, and metabolic diseases were less frequently studied (Figure 1C).

Regardless of the methodology employed to isolate EVs from blood sample of healthy subjects, reported EV concentrations span more than seven orders of magnitude with a geometric mean of $10^{10} \mathrm{EVs}$ per $\mathrm{mL}$ (Figure 2A). This estimate is dependent upon the isolation protocol, although some outlier studies seem to be independent of this, e.g. some very low values measured after ultracentrifugation (Figure $2 \mathrm{~A})$. Those values could largely be explained by the quantification method used to determine the EV concentration, since these methods vary greatly both in their sensitivity and size threshold (Figure 2B).

The reason for the large variation in published data on EV concentrations in plasma is likely multifaceted. The methods used to assess the EV concentration in blood samples are neither specific nor sensitive enough to capture all EVs and/or distinguish EVs from non-EV entities [54]. Also, the EV samples used for enumerations have typically been exposed to purification steps prior to the concentration measurements - steps that likely introduce EV loss and are insufficient in removing all the lipoproteins (discussed below). An inherent large variation of EV concentration in plasma across healthy human individuals has also been reported [23] - a biological variability observed using identical isolation and 
enumeration procedures [9,55]. The variations of several orders of magnitude of the reported EV concentrations in plasma may also be driven by the fact that large variations are easily introduced when studying low abundant entities from a biological specimen (Figure 2). Adding together all of these challenges, we start to understand why there is such a huge discrepancy between the reported EV concentrations in plasma and why we are currently not in a position to determine the exact concentrations of EVs in plasma or any other body fluids, as is possible for other blood components such as lipoproteins [9]. However, we argue that if we consider the majority of concentration measurements derived from nanoparticle tracking analysis (NTA), i.e. keeping the quantification parameter stable, we may close in on the mean EV concentration, hereby yielding an estimate of $\sim 2 * 10^{10} \mathrm{EVs}$ per $\mathrm{mL}$ (Figure $2 \mathrm{C}$ ). The NTA tool is by far the most commonly used technique to determine EV concentrations and size distributions and is currently considered as the gold standard, at least when considering statements from the International Society for Extracellular Vesicles (ISEV) and the extent of use (Figure 2B)[4,54,56-58]. However, NTA does not have the required specificity to distinguish between EVs and the possible non-EV entities contaminating EV isolates from a blood sample, which should be remembered when interpreting this estimate.

\section{Isolation methods and contamination as factors in EV enume ration}

The type of isolation protocol employed to obtain an EV sample is of importance for the outcome of EV concentration measurements. When considering data derived from NTA measurements only (Figure 2C), it is apparent that the least varying data is obtained from ultracentrifugation procedures, providing values close to the geometric mean of all the measurements. Conversely, polyethylene glycol (PEG)-based purification (considered less pure than ultracentrifugation, e.g. ExoQuick ${ }^{\circledR}$ ) and size-exclusion chromatography (SEC)based purification (considered purer than ultracentrifugation) yield datasets with geometric means and variations many-fold higher than those measured for ultracentrifugation techniques. EV concentrations obtained from PEG precipitation is about ten-fold higher than the values based on ultracentrifugation and density gradient ultracentrifugation (Figure 2C). This could be due to the co-precipitation of apoB-containing particles such as chylomicrons and VLDL, IDL and LDL with the EVs [59]. PEG precipitation is actually a well-known method to remove apoB-particles from HDL in plasma samples (Figure 3A)[59,60]. SEC has become a popular method to isolate EVs. However, large apoB-containing lipoproteins (chylomicrons and VLDL, and likely also LDL) are co-eluting with EVs due to their overlapping size distributions (Figure 3B + Figure 4A). Thus, both PEG- and SEC-based isolation methods lead to a significant amount of lipoproteincontamination in the 'purified' EV samples.

One of the limitations of NTA is that it is not capable of distinguishing between EVs and similar sized particles including large lipoproteins [56,61], which could lead to overestimation of the EV concentration in the PEG and SEC-isolated samples due to the significant proportion of large lipoproteins in the EV sample. It is worth mentioning that the biological function of chylomicrons is to deliver dietary lipids from the intestine to the muscle and adipose cells. The chylomicron level thus varies over the course of a day, with the maximum level in blood being reached four hours after a meal, whereby the level drops down after fasting [9]. VLDL levels change in the opposite direction [62]. The relationship between food uptake and chylomicron/VLDL levels may be the reason why reported EV concentrations based on SEC isolation vary so much, clearly stressing the concern about lipoprotein contamination in some of those samples (Figure 2C)[4,9,55,62,63].

The ultracentrifugation method is the most commonly used method to isolate EVs from plasma (or cell cultures)[17,63]. The procedure depends upon the sedimentation rate of differently sized particles with varying densities in a sample, which would suggest that EVs associate with the pellet, whereas the smaller lipoproteins would be present in the supernatant that is most often discarded [4]. The EV concentrations derived from studies employing this technique are much lower than those derived from PEG- 
and SEC-based procedures. For example, there is almost a 15-fold difference between the geometric mean values obtained from SEC and ultracentrifugation, respectively (Figure 2C). While the NTA measurements from this method only fall within two orders of magnitude, there is still problems associated with the purity obtained from this technique (Figure 3C, further discussion below).

When isolating EVs according to their mass density (on a density gradient) instead of size, the contamination of the final EV sample may be reduced. This is because the potential lipoprotein contamination in this procedure (HDL) may theoretically already have been removed in preceding ultracentrifugation steps (according to the gold standard protocols)(Figure $3 \mathrm{C}+\mathrm{D}$ ). However, as we shall see later, the HDL is not likely to be removed completely in this preceding ultracentrifugation step, leaving it able to co-isolate with EVs on a density gradient. Furthermore, NTA is not capable of detecting these fairly small $10 \mathrm{~nm}$ sized HDLs [61,64], and hence, a much lower EV concentration is likely obtained compared to the values derived from the PEG precipitation or SEC purification of EV samples, where larger lipoproteins may contaminate and become detected. The EV concentration deduced from the density gradient ultracentrifugation may therefore provide a good estimate of the EV concentration $\left(\sim 5 * 10^{9} \mathrm{EVs}\right.$ per $\mathrm{mL}$, Figure 2C) when determined using NTA, although the amount of data available on this method is scarce. Also, the impact of high sugar concentration and daylong centrifugation cycles at $>100,000 \mathrm{~g}$ has not been studied in detail, and thus, we know very little about the quality of the isolated sample.

\section{Numbers matter - EVs as a minority population in human blood}

No currently known isolation technique is able to provide pure EV samples due to the significant potential of co-isolating different classes of lipoproteins. This became especially evident with the estimation that EV samples contained at least one order of magnitude higher numbers of LDL particles compared to the EVs $[37,65]$. Furthermore, when platelet-free plasma was subjected to antibody-based pulldown of apoB-48 and apoB-100, the total number of particles measured on the NTA system decreased significantly [66]. Thus, there is little doubt that the number and physicochemical characteristics of lipoproteins are to be considered when wanting to isolate EVs from human blood for studies of their biological function or their potential as disease biomarkers. The different lipoprotein classes are defined according to their density as indicated by their names (Figure 4A). In addition, the apoB-48 protein is uniquely associated with chylomicrons in a 1 to 1 ratio, VLDL, IDL and LDL all contain one apoB-100 protein per particle, while the HDL on average contains three apoA-I protein molecules [9,67]. Taking these stoichiometries into account, the particle concentration of chylomicrons, the other low-density lipoprotein classes (VLDL, IDL and LDL) and HDL can be derived directly from the apoB-48, apoB-100 and apoA-I concentrations in plasma, respectively $[9,67]$. This is in direct opposition to the concentration of EVs, which can only be estimated indirectly due to its more complex and heterogenous structure and composition [55]. The variability of the total apoB and apoA-I concentrations is rather small in healthy individuals compared to EVs - about $30 \%$ and $20 \%$ in terms of relative standard deviations, respectively [68], whereas our data indicate that measurements of the plasma concentration of EVs span 6 - 7 orders of magnitude (Figure 4B).

Determining the concentration of EVs in human blood plasma is difficult, but our data indicates that a value of $\sim 10^{10} \mathrm{EVs}$ per $\mathrm{mL}$ is a relevant estimate (Figure $4 \mathrm{~B}$ ). This value is based on EV concentrations quantified using the NTA technique only and lying between the values obtained for all isolation methods combined $\left(\sim 2 * 10^{10} \mathrm{EVs}\right.$ per $\mathrm{mL}$ - considering the pitfalls of the NTA technique, this may actually illustrate an upper limit of EV concentration in human plasma) and for ultracentrifugation techniques $\left(\sim 5 * 10^{9}\right.$ EVs per $\mathrm{mL}$, least varying data in the dataset). That being said, it does not consider the potential contamination from the lipoproteins as described above (Figure 4A), leading us to the relevant question: Is the EV concentration in plasma high enough compared to the lipoproteins to provide clear cut answers about their roles as biomarkers and the underlying importance this may have on disease biology? If not, how does this affect the interpretations we make on disease biology, when using EVs as biomarkers? 
The total amount of lipoproteins in human plasma ranges around $10^{16}$ particles per $\mathrm{mL}$ (Figure 4C), making the EV the one in a million (information from Simonsen (2017)[9], example of calculation presented in the Supplementary Information). However, when looking at the different types of lipoproteins, their individual concentrations vary significantly (Figure 4C), making some of them less prone to contaminate EV samples using specific isolation methods. For example, chylomicrons are much lower in numbers compared to LDL and HDL particles, whereby they outnumber the EVs by a thousand-fold only. On the other hand, while this is indeed a low concentration compared to HDL particles, the chylomicron concentration will likely still become a severe problem when isolating EVs using SEC. This is further complicated by the indirect methodologies available for EV quantification, making it impossible to decipher how many of the particles measured on the NTA system that could be ascribed to chylomicron contamination, unless the molar concentration of apoB-48 (equal to the chylomicron concentration) was determined in the same samples. One could then go for another isolation method, since EVs and chylomicrons do not have overlapping densities (Figure 4A). The large lipoproteins (chylomicrons and VLDL) can therefore easily be removed from the EV samples by density gradient ultracentrifugation, although this would depict another potential contaminant, namely the HDL particles, which share densities with EVs (Figure 4A)[9]. In the current protocols for density gradient ultracentrifugation, this is rarely considered a problem, since HDL particles are thought to be removed in a prior standard ultracentrifugation step before loading of the density gradient (Figure 3C+D). This is true - in theory - but if we again consider the numeric differences in total HDL versus EV concentration, the problem persists (Figure 4C). Let us do a simple thought experiment: The sedimentation rate $\left(V_{\text {sed }}\right)$ of spherical objects at constant acceleration is proportional to the difference between the density of the particle $\left(\rho_{\text {particle }}\right)$ and the density of the medium $\left(\rho_{\text {medium }}\right)$ and to the square of the particle radius $\left(r_{\text {particle }}^{2}\right): V_{\text {sed }} \propto\left(\rho_{\text {particle }}-\rho_{\text {medium }}\right) r_{\text {particle }}^{2}$. Thus, if we assume that the density of HDL and EVs is similar (Figure 4A), the sedimentation rate of $100 \mathrm{~nm} \mathrm{EVs} \mathrm{is} 100$ times faster than $10 \mathrm{~nm}$ sized HDL particles. If all EVs are sedimented including the ones at the top of a cylindrical vial after a standard 100,000 $\mathrm{g}$ centrifugation step, 1/100 of the HDL particles will co-sediment due to their small but still significant sedimentation rate. Although the HDL contamination has then been reduced about 100 times, the HDL particles will still outnumber the EVs substantially ( $4-5$ orders of magnitude) after the ultracentrifugation step (Figure 4C), especially when also considering that all of the HDL-containing supernatant is rarely removed. Since the HDL particles cannot be detected using the NTA technique, the input from them in EV enumerations may not be counted [61]. This is in principle very positive, because it could yield a more realistic value of the EV concentration. However, for subsequent interpretations made regarding the potential impact the investigated ' $\mathrm{EV}$ ' biomarker may have on the underlying pathophysiology of the disease studied, unknown HDL contamination could have large impact, since these particles are also able to carry specific proteins and RNAs [69-75]. In the case of LDL that is about two times larger than HDL and has a density that is about $10 \%$ less than HDL, the LDL fraction that co-sediment with EVs will be almost similar to that of HDL $(\sim$ 1/100 of the total LDL fraction contained in the samples). A significant LDL contamination was indeed shown when using both ultracentrifugation and density gradient ultracentrifugation [37,65]. Conversely, the lower density of chylomicrons relative to the density of plasma or serum should prevent the chylomicrons to co-sediment with EVs during ultracentrifugation due to their relatively fast buoyancy, meaning that they will flow towards the top of the liquid that is ultracentrifuged. This latter effect may explain why the vast majority of EV concentrations (all except for one) measured after ultracentrifugation or density gradient ultracentrifugation remain below $10^{11}$ EVs per mL (Figure 2C).

To handle these contamination issues, a two-step isolation protocol based on multiple physical parameters (size and density) was proposed to increase the purity of EV samples [9]. Some groups are now applying this two-step strategy to obtain purer EVs samples $[10,76]$. This combination is important if the 
ambition is to increase the overall purity that can be obtained, and the current efforts have shown a significant reduction in the lipoprotein contamination, although the contamination is still fairly high [76]. Surprisingly, a substantial HDL contamination persists after SEC-based isolation (in combination with density gradient ultracentrifugation), which is peculiar given the very large size difference between HDL and EVs [76,77]. Such large differences should be possible to resolve using optimized SEC procedures, which points to the fact that the setup of currently used SEC systems (in-house made or commercially purchased) may be inadequate for true separation to happen [77]. Further optimizing the SEC setup for EV isolation should therefore be possible by introducing an HPLC-like format that would have little difficulties in resolving the different size ranges contained within a plasma sample, e.g. by considering the column type, packing material, flow rate, etc. Also, in relation to handling problems with EV purity, urine-derived EVs have shown great potential to predict prostate cancer in men with a prior negative biopsy [78]. Interestingly, while blood, lymph and cerebrospinal fluids contain significant amounts of lipoproteins, urine contains much less lipoprotein, and could therefore become an alternative way of obtaining the EV biomarkers.

\section{EVs and lipoproteins as biomarkers}

From these examples it seems clear that our current best practice for obtaining EV samples from human blood plasma is challenged by inevitable issues with co-isolating lipoproteins with concentrations that are orders of magnitude higher than what we expect of the EVs. This is the unpleasant truth based on which we have to make our interpretations on studies of EVs isolated from blood plasma, e.g. when evaluated as disease biomarkers. The biomarkers are of increasing interest since they possess the potential of diagnosing devastating cancer diseases at an early timepoint at which curable therapies might still be possible $[3,79]$. The highlighting of EV isolation methods for obtaining the EV biomarkers may seem irrelevant in the sense that whatever was analyzed in a given biomarker study (lipids, proteins, RNA, or DNA) seems to have diagnostic potential [45,79-82]. Can we be sure that these EV biomarkers are in fact EVs? No, we cannot at this moment. This may not necessarily be a problem, if the validity of the biomarker can be ensured by other means. However, in some future uses of EVs in the clinic, specific enumeration may be required.

If diagnostic or therapeutic tools are to be based on a minority population like the EVs, we must be sure that we know what we are analyzing. When using isolated EVs for drug delivery (obtained from blood or serum-containing cell cultures), we must be able to enumerate in great detail to provide a clear value of the therapeutic compound that was dosed to the patient $[2,63,83,84]$. When using the concentration of EVs in the plasma of a patient as a biomarker in itself, the fact that we may be counting mostly lipoproteins could make us blind to what the disease mechanism behind this effect is. When using the cargo contained in the EVs, it is also not possible to completely rule out that lipoproteins may be the particles carrying it $[3,9]$. In the recent years, several reports have described the potential of different lipoproteins to carry proteins and RNAs (small RNAs in particular)[85]. For example, advances in proteomics have extended the list of HDL-associated proteins, beyond the apolipoproteins associated to HDL, to over 85 suggesting that the composition of HDL is more complex than previously anticipated [69]. Studies have also shown that the levels of specific HDL-associated proteins are altered in disease states $[86,87]$ and in polluted environments [88], and could therefore potentially also be used as disease biomarkers [89]. LDL and VLDL are also associated with a growing number of associated proteins that are known to have important functions in dyslipidemia, atherosclerosis, and coagulation disorders [90]. This has important implications on how we interpret EV biomarker data to obtain knowledge about the ongoing disease process, if the protein or RNA message could actually be released and transported by other means than EVs. Moreover, the use of lipidbased disease biomarkers contained in EVs is also highly challenged due to the difficulties in isolating EVs with high purity [3,91,92], because the lipid composition in lipoproteins is very heterogenous and could possibly yield a signal in 'EV' samples [91]. Again, the number of EVs versus lipoproteins could play a big role, since a very small fraction of lipoproteins carrying a lipid, protein, or RNA marker would easily 
outnumber the total number of EVs carrying the same molecule. Our current knowledge on the stoichiometry of RNA-loading into EVs underscores this point, showing that only a very small fraction of EVs are expected to carry a specific type of RNA molecule with a very low copy number [92,93]. This is evident even if specific RNAs are overexpressed in cells to become loaded into EVs [93,94]. On the other hand, there may also be different kinds of molecules that are difficult to imagine in a lipoprotein context, e.g. transmembrane proteins like the tetraspanins, although these proteins may be associated with specific subtypes of EVs only [95]. It could then be possible to avoid overlapping cargoes between EVs and lipoproteins, whereby the robustness of the resulting EV biomarker may increase substantially. This highlights the potential of combining forces between the EV and lipoprotein fields to coordinate efforts and share knowledge to the benefit of the patient.

\section{A continuum of lipid-containing particles}

We are positive that the future is bright for EV biomarkers because their potential is being underscored in the literature with high frequency, and an introduction of these diagnostic tools based on detection of EVs to the clinicians may be coming soon. We provide here an estimate of the plasma EV concentration in healthy subjects of $\sim 10^{10} \mathrm{EVs}$ per mL that may serve as a reference point for future studies characterizing $\mathrm{EV}$ biomarker systems using EV quantification (Figure 4B). The future research into EV-based biomarkers must, however, have a stronger focus on optimizing the isolation protocols to obtain purer samples of EVs, although depending on the type of biomarker, it may be of less importance if it was expressed on EVs or lipoproteins. The higher purity may even yield more robust correlations to any ongoing disease, thereby increasing the clinical potential of the EVs as biomarkers especially in cancer diseases [3,79]. We suggest that new protocols that utilize the multifaceted physicochemical characteristics of EVs could improve the purity of the isolated sample, and therefore also the quality of the resulting EV biomarker, and we believe that the first attempts at using these protocols are encouraging. In this context, the numbers matter a lot. While diagnoses based on EV biomarkers should reach a high throughput stage for proper clinical relevance, there are some methodological issues that cannot be neglected. Thus, the more contamination we accept, the more the EVs will be outnumbered by the lipoproteins. We do not argue against the potential clinical value of the identified EV biomarkers, and it is our belief that EVs are nexuses that mediate a tremendous amount of interesting known and unknown biology that could and should be exploited in a clinical setting. That said, for some biomarker purposes, it may be just as useful to regard the EVs and lipoproteins as a continuum of lipid-containing particles to which biomarker molecules can be associated. Using such a holistic approach, increased strength of plasma-derived cancer biomarkers may soon be revealed.

\section{Acknowledgements}

Frank A.W. Coumans, Laboratory of Experimental Clinical Chemistry, Academic Medical Center, University of Amsterdam, W. Sean Davidson, Pathology and Laboratory Medicine, University of Cincinnati, and Michael C. Phillips, Division of Translational Medicine and Human Genetics, Perelman School of Medicine, University of Pennsylvania are acknowledged for valuable discussions and input to the manuscript. The Novo Nordisk Foundation and the Lundbeck Foundation are gratefully acknowledged for financial support.

\section{References}

[1] S. El Andaloussi, I. Mäger, X.O. Breakefield, M.J.A. Wood, Extracellular vesicles: biology and emerging therapeutic opportunities, Nat Rev Drug Discov. (2013). doi:10.1038/nrd3978.

[2] K.B. Johnsen, J.M. Gudbergsson, M.N. Skov, L. Pilgaard, T. Moos, M. Duroux, A comprehensive overview of exosomes as drug delivery vehicles - endogenous nanocarriers for targeted cancer therapy, Biochim. Biophys. Acta. 1846 (2014) 75-87. doi:10.1016/j.bbcan.2014.04.005.

[3] R. Xu, A. Rai, M. Chen, W. Suwakulsiri, D.W. Greening, R.J. Simpson, Extracellular vesicles in cancer - implications for future improvements in cancer care, Nat Rev Clin Oncol. 15 (2018) 1458. doi:10.1038/s41571-018-0036-9. 
[4] K.W. Witwer, E.I. Buzás, L.T. Bemis, A. Bora, C. Lässer, J. Lötvall, et al., Standardization of sample collection, isolation and analysis methods in extracellular vesicle research, Journal of Extracellular Vesicles. 2 (2013) 20360. doi:10.3402/jev.v2i0.20360.

[5] D. Zocco, P. Ferruzzi, F. Cappello, W.P. Kuo, S. Fais, Extracellular vesicles as shuttles of tumor biomarkers and anti-tumor drugs, Front Oncol. 4 (2014) 267. doi:10.3389/fonc.2014.00267.

[6] S. Fais, L. O'Driscoll, F.E. Borràs, E. Buzas, G. Camussi, F. Cappello, et al., Evidence-Based Clinical Use of Nanoscale Extracellular Vesicles in Nanomedicine, ACS Nano. 10 (2016) 38863899. doi:10.1021/acsnano.5b08015.

[7] F. Cappello, M. Logozzi, C. Campanella, C.C. Bavisotto, A. Marcilla, F. Properzi, et al., Exosome levels in human body fluids: A tumor marker by themselves? Eur J Pharm Sci. 96 (2017) 93-98. doi:10.1016/j.ejps.2016.09.010.

[8] E. van der Pol, A. Sturk, T. van Leeuwen, R. Nieuwland, F. Coumans, the ISTH-SSC-VB Working group, Standardization of extracellular vesicle measurements by flow cytometry through vesicle diameter approximation, J. Thromb. Haemost. 16 (2018) 1236-1245. doi:10.1111/jth.14009.

[9] J.B. Simonsen, What Are We Looking At? Extracellular Vesicles, Lipoproteins, or Both? Circ. Res. 121 (2017) 920-922. doi:10.1161/CIRCRESAHA.117.311767.

[10] L. Muller, C.-S. Hong, D.B. Stolz, S.C. Watkins, T.L. Whiteside, Isolation of biologically-active exosomes from human plasma, J. Immunol. Methods. 411 (2014) 55-65.

doi:10.1016/j.jim.2014.06.007.

[11] M. Verma, T.K. Lam, E. Hebert, R.L. Divi, Extracellular vesicles: potential applications in cancer diagnosis, prognosis, and epidemiology, BMC Clin Pathol. 15 (2015) 14. doi:10.1186/s12907-0150005-5.

[12] I. Parolini, C. Federici, C. Raggi, L. Lugini, S. Palleschi, A. De Milito, et al., Microenvironmental $\mathrm{pH}$ is a key factor for exosome traffic in tumor cells, J. Biol. Chem. 284 (2009) 34211-34222. doi:10.1074/jbc.M109.041152.

[13] M. Logozzi, D.F. Angelini, E. Iessi, D. Mizzoni, R. Di Raimo, C. Federici, et al., Increased PSA expression on prostate cancer exosomes in in vitro condition and in cancer patients, Cancer Letters. 403 (2017) 318-329. doi:10.1016/j.canlet.2017.06.036.

[14] E. Spugnini, S. Fais, Proton pump inhibition and cancer therapeutics: A specific tumor targeting or it is a phenomenon secondary to a systemic buffering? Semin. Cancer Biol. 43 (2017) 111-118. doi:10.1016/j.semcancer.2017.01.003.

[15] S. Fais, G. Venturi, B. Gatenby, Microenvironmental acidosis in carcinogenesis and metastases: new strategies in prevention and therapy, Cancer Metastasis Rev. 33 (2014) 1095-1108. doi:10.1007/s10555-014-9531-3.

[16] M. Logozzi, D. Mizzoni, D.F. Angelini, R. Di Raimo, M. Falchi, L. Battistini, et al., Microenvironmental pH and Exosome Levels Interplay in Human Cancer Cell Lines of Different Histotypes, Cancers (Basel). 10 (2018) 370. doi:10.3390/cancers 10100370.

[17] EV-TRACK Consortium, J. Van Deun, P. Mestdagh, P. Agostinis, Ö. Akay, S. Anand, et al., EVTRACK: transparent reporting and centralizing knowledge in extracellular vesicle research, Nat. Methods. 14 (2017) 228-232. doi:10.1038/nmeth.4185.

[18] C. Liu, X. Xu, B. Li, B. Situ, W. Pan, Y. Hu, et al., Single-Exosome-Counting Immunoassays for Cancer Diagnostics, Nano Lett. 18 (2018) 4226-4232. doi:10.1021/acs.nanolett.8b01184.

[19] D. Buschmann, B. Kirchner, S. Hermann, M. Märte, C. Wurmser, F. Brandes, et al., Evaluation of serum extracellular vesicle isolation methods for profiling miRNAs by next-generation sequencing, Journal of Extracellular Vesicles. 7 (2018) 1481321. doi:10.1080/20013078.2018.1481321.

[20] T. Soares Martins, J. Catita, I. Martins Rosa, O. A B da Cruz E Silva, A.G. Henriques, Exosome isolation from distinct biofluids using precipitation and column-based approaches, PLoS ONE. 13 (2018) e0198820. doi:10.1371/journal.pone.0198820.

[21] R. Stranska, L. Gysbrechts, J. Wouters, P. Vermeersch, K. Bloch, D. Dierickx, et al., Comparison of membrane affinity-based method with size-exclusion chromatography for isolation of exosome-like vesicles from human plasma, J Transl Med. 16 (2018) 1. doi:10.1186/s12967-017-1374-6. 
[22] Y. Xu, X. Ku, C. Wu, C. Cai, J. Tang, W. Yan, Exosomal proteome analysis of human plasma to monitor sepsis progression, Biochem. Biophys. Res. Commun. 499 (2018) 856-861. doi:10.1016/j.bbrc.2018.04.006.

[23] S.J. Kuravi, C.M. Yates, M. Foster, P. Harrison, J. Hazeldine, P. Hampson, et al., Changes in the pattern of plasma extracellular vesicles after severe trauma, PLoS ONE. 12 (2017) e0183640. doi:10.1371/journal.pone.0183640.

[24] Y.-T. Kang, Y.J. Kim, J. Bu, Y.-H. Cho, S.-W. Han, B.-I. Moon, High-purity capture and release of circulating exosomes using an exosome-specific dual-patterned immunofiltration (ExoDIF) device, Nanoscale. 9 (2017) 13495-13505. doi:10.1039/c7nr04557c.

[25] M. Wu, Y. Ouyang, Z. Wang, R. Zhang, P.-H. Huang, C. Chen, et al., Isolation of exosomes from whole blood by integrating acoustics and microfluidics, Proc. Natl. Acad. Sci. U.S.a. 114 (2017) 10584-10589. doi:10.1073/pnas.1709210114.

[26] Y.-E. Cho, E. Mezey, J.P. Hardwick, N. Salem, D.L. Clemens, B.-J. Song, Increased ethanolinducible cytochrome P450-2E1 and cytochrome P450 isoforms in exosomes of alcohol-exposed rodents and patients with alcoholism through oxidative and endoplasmic reticulum stress, Hepatol Commun. 1 (2017) 675-690. doi:10.1002/hep4.1066.

[27] M. Garcia-Contreras, S.H. Shah, A. Tamayo, P.D. Robbins, R.B. Golberg, A.J. Mendez, et al., Plasma-derived exosome characterization reveals a distinct microRNA signature in long duration Type 1 diabetes, Sci Rep. 7 (2017) 5998. doi:10.1038/s41598-017-05787-y.

[28] M.R. Fernando, C. Jiang, G.D. Krzyzanowski, W.L. Ryan, New evidence that a large proportion of human blood plasma cell-free DNA is localized in exosomes, PLoS ONE. 12 (2017) e0183915. doi:10.1371/journal.pone.0183915.

[29] S. Ludwig, T. Floros, M.-N. Theodoraki, C.-S. Hong, E.K. Jackson, S. Lang, et al., Suppression of Lymphocyte Functions by Plasma Exosomes Correlates with Disease Activity in Patients with Head and Neck Cancer, Clin. Cancer Res. 23 (2017) 4843-4854. doi:10.1158/1078-0432.CCR-16-2819.

[30] W. Ye, X. Tang, Z. Yang, C. Liu, X. Zhang, J. Jin, et al., Plasma-derived exosomes contribute to inflammation via the TLR9-NF- $\kappa B$ pathway in chronic heart failure patients, Mol. Immunol. 87 (2017) 114-121. doi:10.1016/j.molimm.2017.03.011.

[31] T. Thomou, M.A. Mori, J.M. Dreyfuss, M. Konishi, M. Sakaguchi, C. Wolfrum, et al., Adiposederived circulating miRNAs regulate gene expression in other tissues, Nature. 542 (2017) 450-455. doi:10.1038/nature21365.

[32] P.J. Wermuth, S. Piera-Velazquez, S.A. Jimenez, Exosomes isolated from serum of systemic sclerosis patients display alterations in their content of profibrotic and antifibrotic microRNA and induce a profibrotic phenotype in cultured normal dermal fibroblasts, Clin. Exp. Rheumatol. 35 Suppl 106 (2017) 21-30.

[33] K. Allenson, J. Castillo, F.A. San Lucas, G. Scelo, D.U. Kim, V. Bernard, et al., High prevalence of mutant KRAS in circulating exosome-derived DNA from early-stage pancreatic cancer patients, Ann. Oncol. 28 (2017) 741-747. doi:10.1093/annonc/mdx004.

[34] Y. Matsumoto, M. Kano, Y. Akutsu, N. Hanari, I. Hoshino, K. Murakami, et al., Quantification of plasma exosome is a potential prognostic marker for esophageal squamous cell carcinoma, Oncol. Rep. 36 (2016) 2535-2543. doi:10.3892/or.2016.5066.

[35] J.Y. Lee, J.K. Park, E.Y. Lee, E.B. Lee, Y.W. Song, Circulating exosomes from patients with systemic lupus erythematosus induce an proinflammatory immune response, Arthritis Res. Ther. 18 (2016) 264. doi:10.1186/s13075-016-1159-y.

[36] C. Krafft, K. Wilhelm, A. Eremin, S. Nestel, N. von Bubnoff, W. Schultze-Seemann, et al., A specific spectral signature of serum and plasma-derived extracellular vesicles for cancer screening, Nanomedicine. 13 (2017) 835-841. doi:10.1016/j.nano.2016.11.016.

[37] B.W. Sódar, A. Kittel, K. Pálóczi, K.V. Vukman, X. Osteikoetxea, K. Szabó-Taylor, et al., Lowdensity lipoprotein mimics blood plasma-derived exosomes and microvesicles during isolation and detection, Sci Rep. 6 (2016) 24316. doi:10.1038/srep24316.

[38] A. Alberro, M. Sáenz-Cuesta, M. Muñoz-Culla, M. Mateo-Abad, E. Gonzalez, E. Carrasco-Garcia, et al., Inflammaging and Frailty Status Do Not Result in an Increased Extracellular Vesicle Concentration in Circulation, Int J Mol Sci. 17 (2016) 1168. doi:10.3390/ijms17071168. 
[39] K. Schara, R. Štukelj, J.L. Krek, K. Lakota, S. Sodin-Šemrl, A.J.M. Boulton, et al., A study of extracellular vesicle concentration in active diabetic Charcot neuroarthropathy, Eur J Pharm Sci. 98 (2017) 58-63. doi:10.1016/j.ejps.2016.09.009.

[40] E.J. Goetzl, L. Goetzl, J.S. Karliner, N. Tang, L. Pulliam, Human plasma platelet-derived exosomes: effects of aspirin, Faseb J. 30 (2016) 2058-2063. doi:10.1096/fj.201500150R.

[41] C.-S. Hong, S. Funk, L. Muller, M. Boyiadzis, T.L. Whiteside, Isolation of biologically active and morphologically intact exosomes from plasma of patients with cancer, Journal of Extracellular Vesicles. 5 (2016) 29289. doi:10.3402/jev.v5.29289.

[42] M.A.J. van Eijndhoven, J.M. Zijlstra, N.J. Groenewegen, E.E.E. Drees, S. van Niele, S.R. Baglio, et al., Plasma vesicle miRNAs for therapy response monitoring in Hodgkin lymphoma patients, JCI Insight. 1 (2016) e89631. doi:10.1172/jci.insight.89631.

[43] Y. Yuana, A.N. Böing, A.E. Grootemaat, E. van der Pol, C.M. Hau, P. Cizmar, et al., Handling and storage of human body fluids for analysis of extracellular vesicles, Journal of Extracellular Vesicles. 4 (2015) 29260. doi:10.3402/jev.v4.29260.

[44] T. Baranyai, K. Herczeg, Z. Onódi, I. Voszka, K. Módos, N. Marton, et al., Isolation of Exosomes from Blood Plasma: Qualitative and Quantitative Comparison of Ultracentrifugation and Size Exclusion Chromatography Methods, PLoS ONE. 10 (2015) e0145686. doi:10.1371/journal.pone.0145686.

[45] S.A. Melo, L.B. Luecke, C. Kahlert, A.F. Fernandez, S.T. Gammon, J. Kaye, et al, Glypican-1 identifies cancer exosomes and detects early pancreatic cancer, Nature. 523 (2015) 177-182. doi:10.1038/nature 14581.

[46] R.J. Lobb, M. Becker, S.W. Wen, C.S.F. Wong, A.P. Wiegmans, A. Leimgruber, et al, Optimized exosome isolation protocol for cell culture supernatant and human plasma, Journal of Extracellular Vesicles. 4 (2015) 27031. doi:10.3402/jev.v4.27031.

[47] F. Momen-Heravi, B. Saha, K. Kodys, D. Catalano, A. Satishchandran, G. Szabo, Increased number of circulating exosomes and their microRNA cargos are potential novel biomarkers in alcoholic hepatitis, J Transl Med. 13 (2015) 261. doi:10.1186/s12967-015-0623-9.

[48] D. Enderle, A. Spiel, C.M. Coticchia, E. Berghoff, R. Mueller, M. Schlumpberger, et al., Characterization of RNA from Exosomes and Other Extracellular Vesicles Isolated by a Novel Spin Column-Based Method, PLoS ONE. 10 (2015) e0136133. doi:10.1371/journal.pone.0136133.

[49] M. Li, E. Zeringer, T. Barta, J. Schageman, A. Cheng, A.V. Vlassov, Analysis of the RNA content of the exosomes derived from blood serum and urine and its potential as biomarkers, Philos. Trans. R. Soc. Lond., B, Biol. Sci. 369 (2014) 20130502-20130502. doi:10.1098/rstb.2013.0502.

[50] J.R. Chevillet, Q. Kang, I.K. Ruf, H.A. Briggs, L.N. Vojtech, S.M. Hughes, et al., Quantitative and stoichiometric analysis of the microRNA content of exosomes, Proc. Natl. Acad. Sci. U.S.a. 111 (2014) 14888-14893. doi:10.1073/pnas.1408301111.

[51] J. Caradec, G. Kharmate, E. Hosseini-Beheshti, H. Adomat, M. Gleave, E. Guns, Reproducibility and efficiency of serum-derived exosome extraction methods, Clin. Biochem. 47 (2014) 12861292. doi:10.1016/j.clinbiochem.2014.06.011.

[52] J. Schageman, E. Zeringer, M. Li, T. Barta, K. Lea, J. Gu, et al., The complete exosome workflow solution: from isolation to characterization of RNA cargo, Biomed Res Int. 2013 (2013) 25395715. doi:10.1155/2013/253957.

[53] R.A. Dragovic, J.H. Southcombe, D.S. Tannetta, C.W.G. Redman, I.L. Sargent, Multicolor flow cytometry and nanoparticle tracking analysis of extracellular vesicles in the plasma of normal pregnant and pre-eclamptic women, Biol. Reprod. 89 (2013) 151. doi:10.1095/biolreprod.113.113266.

[54] J. Lötvall, A.F. Hill, F. Hochberg, E.I. Buzás, D. Di Vizio, C. Gardiner, et al., Minimal experimental requirements for definition of extracellular vesicles and their functions: a position statement from the International Society for Extracellular Vesicles, Journal of Extracellular Vesicles. 3 (2014) 26913. doi:10.3402/jev.v3.26913.

[55] F.A.W. Coumans, A.R. Brisson, E.I. Buzás, F. Dignat-George, E.E.E. Drees, S. El-Andaloussi, et al., Methodological Guidelines to Study Extracellular Vesicles, Circ. Res. 120 (2017) 1632-1648. doi:10.1161/CIRCRESAHA.117.309417. 
[56] C. Gardiner, Y.J. Ferreira, R.A. Dragovic, C.W.G. Redman, I.L. Sargent, Extracellular vesicle sizing and enumeration by nanoparticle tracking analysis, Journal of Extracellular Vesicles. 2 (2013) 19671. doi:10.3402/jev.v2i0.19671.

[57] T. Lener, M. Gimona, L. Aigner, V. Börger, E. Buzas, G. Camussi, et al., Applying extracellular vesicles based therapeutics in clinical trials - an ISEV position paper, Journal of Extracellular Vesicles. 4 (2015) 30087.

[58] M. Yáñez-Mó, P.R.-M. Siljander, Z. Andreu, A.B. Zavec, F.E. Borràs, E.I. Buzás, et al., Biological properties of extracellular vesicles and their physiological functions, Journal of Extracellular Vesicles. 4 (2015) 27066. doi:10.3402/jev.v4.27066.

[59] W.S. Davidson, A. Heink, H. Sexmith, J.T. Melchior, S.M. Gordon, Z. Kuklenyik, et al., The effects of apolipoprotein B depletion on HDL subspecies composition and function, J. Lipid Res. 57 (2016) 674-686. doi:10.1194/jlr.M066613.

[60] P.H. Iverius, T.C. Laurent, Precipitation of some plasma proteins by the addition of dextran or polyethylene glycol, Biochim. Biophys. Acta. 133 (1967) 371-373.

[61] R.A. Dragovic, C. Gardiner, A.S. Brooks, D.S. Tannetta, D.J.P. Ferguson, P. Hole, et al., Sizing and phenotyping of cellular vesicles using Nanoparticle Tracking Analysis, Nanomedicine. 7 (2011) 780-788. doi:10.1016/j.nano.2011.04.003.

[62] B.G. Nordestgaard, M. Benn, Fasting and nonfasting LDL cholesterol: to measure or calculate? Clinical Chemistry. 55 (2009) 845-847. doi:10.1373/clinchem.2008.123083.

[63] J.M. Gudbergsson, K.B. Johnsen, M.N. Skov, M. Duroux, Systematic review of factors influencing extracellular vesicle yield from cell cultures, Cytotechnology. 68 (2016) 579-592. doi:10.1007/s10616-015-9913-6.

[64] V. Filipe, A. Hawe, W. Jiskoot, Critical evaluation of Nanoparticle Tracking Analysis (NTA) by NanoSight for the measurement of nanoparticles and protein aggregates, Pharm. Res. 27 (2010) 796-810. doi:10.1007/s11095-010-0073-2.

[65] Y. Yuana, J. Levels, A. Grootemaat, A. Sturk, R. Nieuwland, Co-isolation of extracellular vesicles and high-density lipoproteins using density gradient ultracentrifugation, Journal of Extracellular Vesicles. 3 (2014) 23262. doi:10.3402/jev.v3.23262.

[66] M. Mørk, A. Handberg, S. Pedersen, M.M. Jørgensen, R. Bæk, M.K. Nielsen, et al., Prospects and limitations of antibody-mediated clearing of lipoproteins from blood plasma prior to nanoparticle tracking analysis of extracellular vesicles, Journal of Extracellular Vesicles. 6 (2017) 1308779. doi:10.1080/20013078.2017.1308779.

[67] A. Jones, M.C. Phillips, Lipoprotein structure, in: D.E. Vance, J.E. Vance (Eds.), Biochemistry of Lipids, Lipoproteins and Membranes, Elsevier, 2008. doi:10.1016/B978-0-444-53219-0. X5001-6.

[68] A. Gille, D. D’Andrea, M.A. Tortorici, G. Hartel, S.D. Wright, CSL112 (Apolipoprotein A-I [Human]) Enhances Cholesterol Efflux Similarly in Healthy Individuals and Stable Atherosclerotic Disease Patients, Arterioscler. Thromb. Vasc. Biol. 38 (2018) 953-963. doi:10.1161/ATVBAHA.118.310538.

[69] A.S. Shah, L. Tan, J.L. Long, W.S. Davidson, Proteomic diversity of high density lipoproteins: our emerging understanding of its importance in lipid transport and beyond, J. Lipid Res. 54 (2013) 2575-2585. doi:10.1194/jlr.R035725.

[70] K.C. Vickers, B.T. Palmisano, B.M. Shoucri, R.D. Shamburek, A.T. Remaley, MicroRNAs are transported in plasma and delivered to recipient cells by high-density lipoproteins, Nat Cell Biol. 13 (2011) 423-433. doi:10.1038/ncb2210.

[71] K.C. Vickers, A.T. Remaley, Lipid-based carriers of microRNAs and intercellular communication, Current Opinion in Lipidology. 23 (2012) 91-97. doi:10.1097/MOL.0b013e328350a425.

[72] K.C. Vickers, K.-A. Rye, F. Tabet, microRNAs in the onset and development of cardiovascular disease, Clin. Sci. 126 (2014) 183-194. doi:10.1042/CS20130203.

[73] F. Tabet, K.C. Vickers, L.F. Cuesta Torres, C.B. Wiese, B.M. Shoucri, G. Lambert, et al, HDLtransferred microRNA-223 regulates ICAM-1 expression in endothelial cells, Nat Commun. 5 (2014) 3292. doi:10.1038/ncomms4292. 
[74] D.L. Michell, R.M. Allen, S.R. Landstreet, S. Zhao, C.L. Toth, Q. Sheng, et al., Isolation of Highdensity Lipoproteins for Non-coding Small RNA Quantification, J Vis Exp. (2016). doi:10.3791/54488.

[75] J. Wagner, M. Riwanto, C. Besler, A. Knau, S. Fichtlscherer, T. Röxe, et al., Characterization of levels and cellular transfer of circulating lipoprotein-bound microRNAs, Arterioscler. Thromb. Vasc. Biol. 33 (2013) 1392-1400. doi:10.1161/ATVBAHA.112.300741.

[76] N. Karimi, A. Cvjetkovic, S.C. Jang, R. Crescitelli, M.A. Hosseinpour Feizi, R. Nieuwland, et al., Detailed analysis of the plasma extracellular vesicle proteome after separation from lipoproteins, Cell. Mol. Life Sci. 75 (2018) 2873-2886. doi:10.1007/s00018-018-2773-4.

[77] A.N. Böing, E. van der Pol, A.E. Grootemaat, F.A.W. Coumans, A. Sturk, R. Nieuwland, Singlestep isolation of extracellular vesicles by size-exclusion chromatography, Journal of Extracellular Vesicles. 3 (2014) 23430. doi:10.3402/jev.v3.23430.

[78] M.J. Donovan, P. Torkler, M. Noerholm, J. Skog, J.M. McKiernan, Performance of a clinically validated urine exosome gene expression test to predict high grade prostate cancer in men with a prior negative biopsy, J. Clin. Oncol. 36 (2018) 119-119. doi:10.1200/JCO.2018.36.6_suppl.119.

[79] S. Anfossi, A. Babayan, K. Pantel, G.A. Calin, Clinical utility of circulating non-coding RNAs an update, Nat Rev Clin Oncol. 19 (2018) 1423. doi:10.1038/s41571-018-0035-x.

[80] K.S. Yang, H. Im, S. Hong, I. Pergolini, A.F. del Castillo, R. Wang, et al., Multiparametric plasma EV profiling facilitates diagnosis of pancreatic malignancy, Sci Trans1 Med. 9 (2017) eaal3226. doi:10.1126/scitranslmed.aal3226.

[81] H. Schwarzenbach, N. Nishida, G.A. Calin, K. Pantel, Clinical relevance of circulating cell-free microRNAs in cancer, Nat Rev Clin Oncol. 11 (2014) 145-156. doi:10.1038/nrclinonc.2014.5.

[82] G. Siravegna, S. Marsoni, S. Siena, A. Bardelli, Integrating liquid biopsies into the management of cancer, Nat Rev Clin Oncol. 14 (2017) 531-548. doi:10.1038/nrclinonc.2017.14.

[83] N. Humle, K.B. Johnsen, G.A. Arendt, R.P. Nielsen, T. Moos, L.B. Thomsen, Targeted Vascular Drug Delivery in Cerebral Cancer, Curr. Pharm. Des. 22 (2016) 5487-5504.

[84] K.B. Johnsen, J.M. Gudbergsson, M. Duroux, T. Moos, T.L. Andresen, J.B. Simonsen, On the use of liposome controls in studies investigating the clinical potential of extracellular vesicle-based drug delivery systems - A commentary, Journal of Controlled Release. (2017). doi:10.1016/j.jconrel.2017.11.002.

[85] R.M. Allen, S. Zhao, M.A. Ramirez-Solano, W. Zhu, D.L. Michell, Y. Wang, et al., Bioinformatic analysis of endogenous and exogenous small RNAs on lipoproteins, Journal of Extracellular Vesicles. 7 (2018) 1506198. doi:10.1080/20013078.2018.1506198.

[86] J.E. Emmens, D.J.L. Jones, T.H. Cao, D.C.S. Chan, S.P.R. Romaine, P.A. Quinn, et al., Proteomic diversity of high-density lipoprotein explains its association with clinical outcome in patients with heart failure, Eur J Heart Fail. 20 (2017) 260-267. doi:10.1002/ejhf.1101.

[87] E. Burillo, I. Jorge, D. Martínez-López, E. Camafeita, L.M. Blanco-Colio, M. Trevisan-Herraz, et al., Quantitative HDL Proteomics Identifies Peroxiredoxin-6 as a Biomarker of Human Abdominal Aortic Aneurysm, Sci Rep. 6 (2016) 1125. doi:10.1038/srep38477.

[88] S.A. Ljunggren, I. Helmfrid, U. Norinder, M. Fredriksson, G. Wingren, H. Karlsson, et al., Alterations in high-density lipoprotein proteome and function associated with persistent organic pollutants, Environment International. 98 (2017) 204-211. doi:10.1016/j.envint.2016.11.008.

[89] A. von Zychlinski, T. Kleffmann, Dissecting the proteome of lipoproteins: New biomarkers for cardiovascular diseases? Translational Proteomics. 7 (2015) 30-39. doi:10.1016/j.trprot.2014.12.001.

[90] M. Dashty, M. Motazacker, J. Levels, M. de Vries, M. Mahmoudi, M. Peppelenbosch, et al., Proteome of human plasma very low-density lipoprotein and low-density lipoprotein exhibits a link with coagulation and lipid metabolism, Thromb Haemost. 111 (2017) 518-530. doi:10.1160/TH1302-0178.

[91] N. Christinat, M. Masoodi, Comprehensive Lipoprotein Characterization Using Lipidomics Analysis of Human Plasma, J. Proteome Res. 16 (2017) 2947-2953. doi:10.1021/acs.jproteome.7b00236. 
[92] S.W. Ferguson, J. Nguyen, Exosomes as therapeutics: The implications of molecular composition and exosomal heterogeneity, J Control Release. 228 (2016) 179-190. doi:10.1016/j.jconrel.2016.02.037.

[93] D.S. Sutaria, M. Badawi, M.A. Phelps, T.D. Schmittgen, Achieving the Promise of Therapeutic Extracellular Vesicles: The Devil is in Details of Therapeutic Loading, Pharm. Res. 34 (2017) 1053-1066. doi:10.1007/s11095-017-2123-5.

[94] D.S. Sutaria, J. Jiang, O.A. Elgamal, S.M. Pomeroy, M. Badawi, X. Zhu, et al., Low active loading of cargo into engineered extracellular vesicles results in inefficient miRNA mimic delivery, Journal of Extracellular Vesicles. 6 (2017) 1333882.

[95] J. Kowal, G. Arras, M. Colombo, M. Jouve, J.P. Morath, B. Primdal-Bengtson, et al., Proteomic comparison defines novel markers to characterize heterogeneous populations of extracellular vesicle subtypes, Proc. Natl. Acad. Sci. U.S.a. 113 (2016) E968-77. doi:10.1073/pnas.1521230113.

Figure 1. Extracellular vesicles as biomarkers of disease. (A) Extracellular vesicles (EVs) are a diverse family of lipid bilayer particles that are secreted by all cells of the body. They are composed of many different lipids (especially phospholipids and cholesterol), proteins (especially tetraspanin proteins), and nucleic acids. EVs are important for the intercellular communication, in which cells share different kinds of information with each other, either in a paracrine fashion to its immediate neighbors, or over large distances, where the EVs transport via the systemic circulation. (B) Publication frequency of studies investigating EVs in human blood. Data was derived from three different PubMed searches; (exosome OR "extracellular vesicle") $A N D X$ AND year, where $X$ is explained in the figure. (C) Thematic analysis of the all EV biomarker studies from the search "(exosome OR "extracellular vesicle") AND (plasma OR blood) AND year" between $2013-2018$.

Figure 2. Extracellular vesicle concentration in human blood. Estimates of the extracellular vesicle (EV) concentration in human blood was derived from the PubMed search "(exosome OR "extracellular vesicle") AND (plasma OR blood) AND human AND year" between 2013 - 2018, i.e. the period with the sharpest increase in publication frequency. Detailed analysis of the search outcome yielded 37 studies that provided 58 estimates of the EV concentration in blood from healthy human subjects. Estimates from subjects with disease or from other species were not included. (A) The plasma EV concentration measured in humans span more than 7 orders of magnitude with a geometric mean of approximately $\sim 10^{10} \mathrm{EV}$ per $\mathrm{mL}$. Subanalysis revealed a substantial difference between the estimates derived from different isolation techniques, although with some intergroup variation as well. (B) When the quantification methodology was considered, flow cytometry was found to severely underestimate the EV concentration when compared to other techniques including the gold standard method, NTA. (C) Outlier estimates could largely be removed by considering estimates derived from nanoparticle tracking analysis only, after which the geometric mean of the EV concentration in human blood was found to be $\sim 2 * 10^{10} \mathrm{EVs}$ per mL. EV: Extracellular vesicle. SEC: Size exclusion chromatography. PEG: Polyethylene glycol-based precipitation. UC: Ultracentrifugation. DG: Density gradient ultracentrifugation. NTA: Nanoparticle tracking analysis. TRPS: Tunable resistive pulse sensing. FC: Flow cytometry. AEA: Acetylcholine esterase activity.

Figure 3. Methods for isolating extracellular vesicles. Extracellular vesicles (EVs) can be isolated using a variety of techniques depending on their specific physicochemical properties, most prominently the size and density. All currently used isolation methods are prone to lipoprotein contamination, although with differences as to which lipoproteins that will co-isolate and with varying impact. (A) Volume-excluding techniques such as polyethylene glycol (PEG)-based precipitation is a fast method for obtaining EV samples. The principle behind the technique is to mix a biological sample, e.g. plasma, with a specific amount of PEG-containing solution (black strands), followed by centrifugation at approximately $10,000 \mathrm{~g}$. The method is a classical way of purifying HDL particles in the supernatant, and therefore, LDL, VLDL, and chylomicrons will likely co-isolate in the EV-containing pellet after centrifugation. (B) Size-exclusion 
chromatography purifies particles in a solution as a function of their hydrodynamic diameter. The biological sample is passed through a column, wherein smaller particles (e.g. HDL) are slowed and larger particles (e.g. EVs) move freely through the column material. Due to the similarity in size distribution between EVs and chylomicrons, these two types of particles will likely co-isolate in the void fraction. (C) Ultracentrifugation is the most widely used isolation method, and its isolation capacity is based on pelleting the EVs at centrifugation speeds $>100,000 \mathrm{~g}$. Due to the low number of EVs in a plasma sample compared to the different lipoproteins, a significant number of lipoproteins may be contained in the resulting pellet. This is depicted by the sedimentation rate and concentration of each type of lipoprotein and EVs. Given the low density of chylomicrons, these particles will likely move upwards instead of downwards to the pellet during ultracentrifugation. (D) In density gradient ultracentrifugation, the difference particles contained in the plasma sample are isolated based on their density. This means that HDL (and LDL) particles likely co-isolate with EVs, if these contaminants are not removed by other means. PEG: Polyethylene glycol. VLDL: Very low-density lipoprotein. LDL: Low-density lipoprotein. HDL: High-density lipoprotein.

Figure 4. Extracellular vesicles as a minority population in human plasma. (A) Extracellular vesicles (EVs) share several physicochemical properties with the different lipoprotein particles contained in the plasma. For example, the size distribution is very similar to chylomicrons, whereas the density distribution is similar to that of HDL. The figure was modified from Simonsen (2017) [9]. (B) Estimation of the EV concentration in human plasma based on data obtained from studies using nanoparticles tracking analysis as a quantification method. The geometric mean of the dataset suggests that the EV concentration in human plasma is $\sim 2 * 10^{10} \mathrm{EVs}$ per $\mathrm{mL}$. (C) Particle concentration of lipoproteins and EVs in human plasma. EVs are outnumbered in human plasma by 6 orders of magnitude higher total concentration of the different lipoproteins. The VLDL concentration is not depicted since the majority of apoB-100 expression (present on VLDL) in human plasma can be ascribed to LDL particles. Lipoprotein particle concentrations were obtained from Simonsen (2017) [9]. 
A

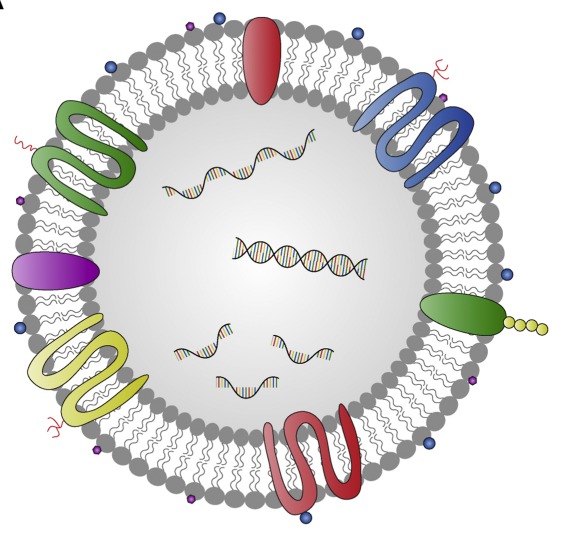

\section{B}

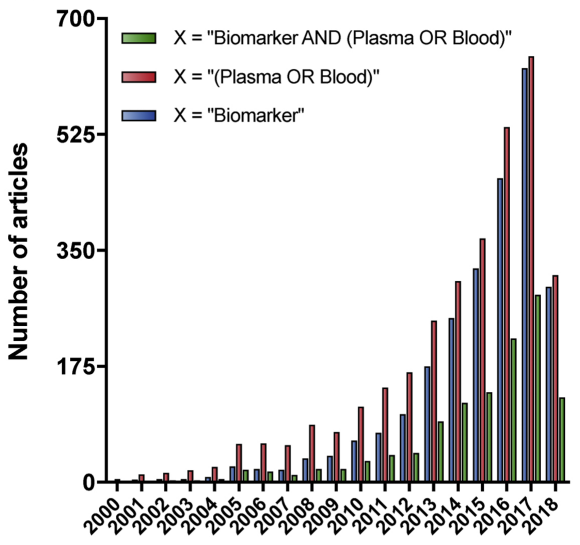

C

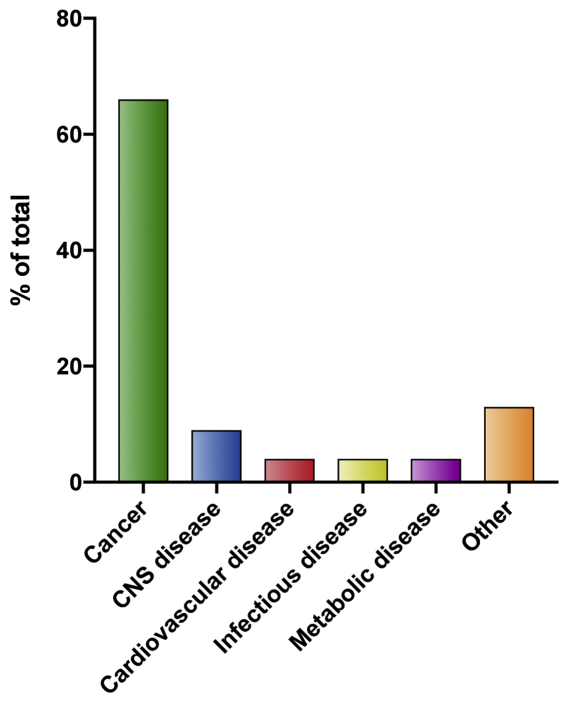


A Isolation method

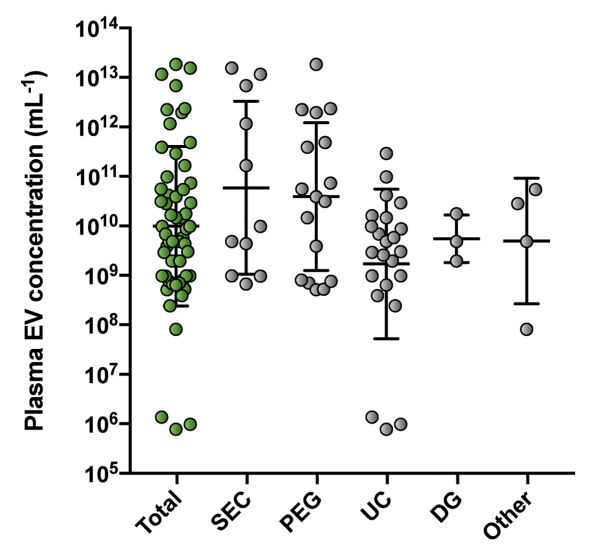

B

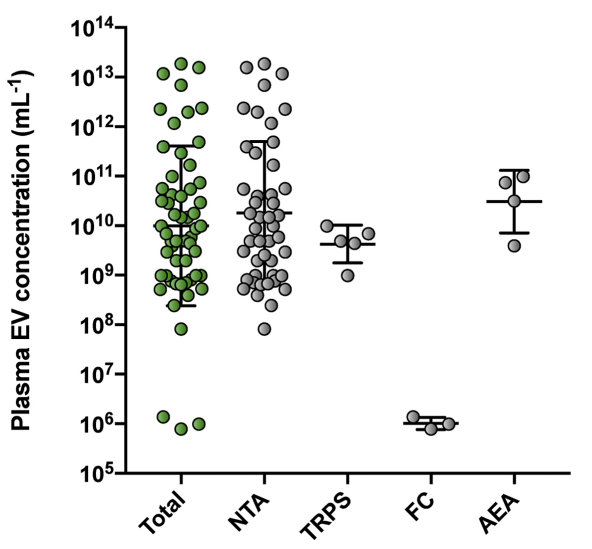

C Isolation method (NTA only)

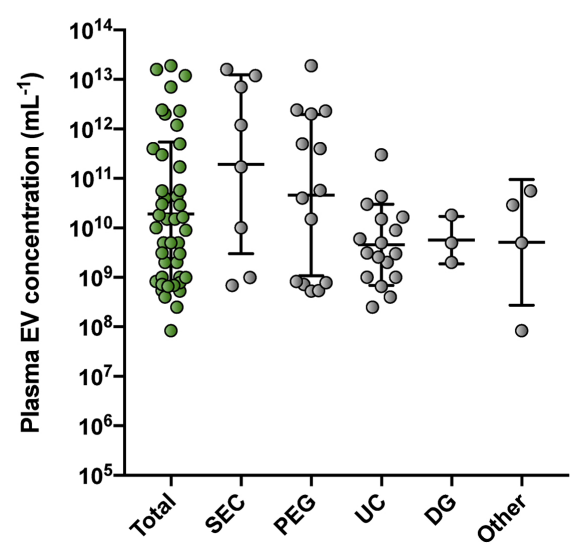

\section{Figure 2}


A

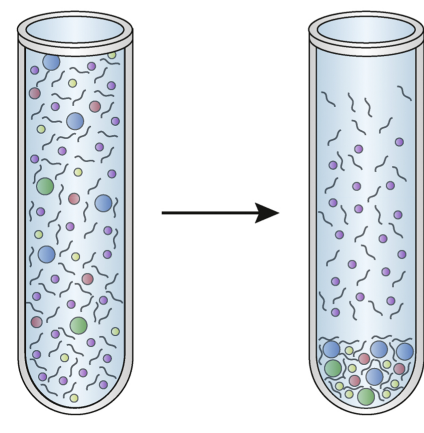

B

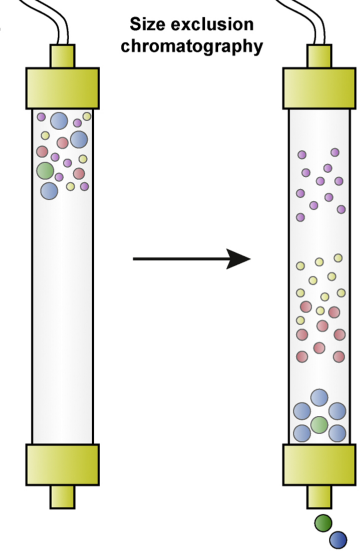

C

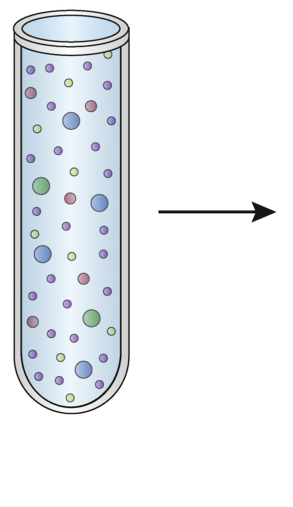

Ultracentrifugation

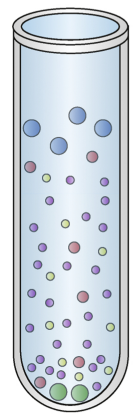

D Density gradient ultracentrifugation

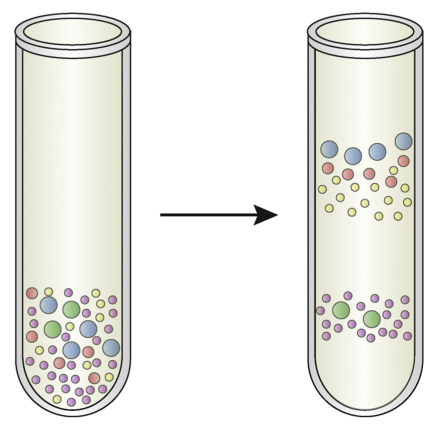



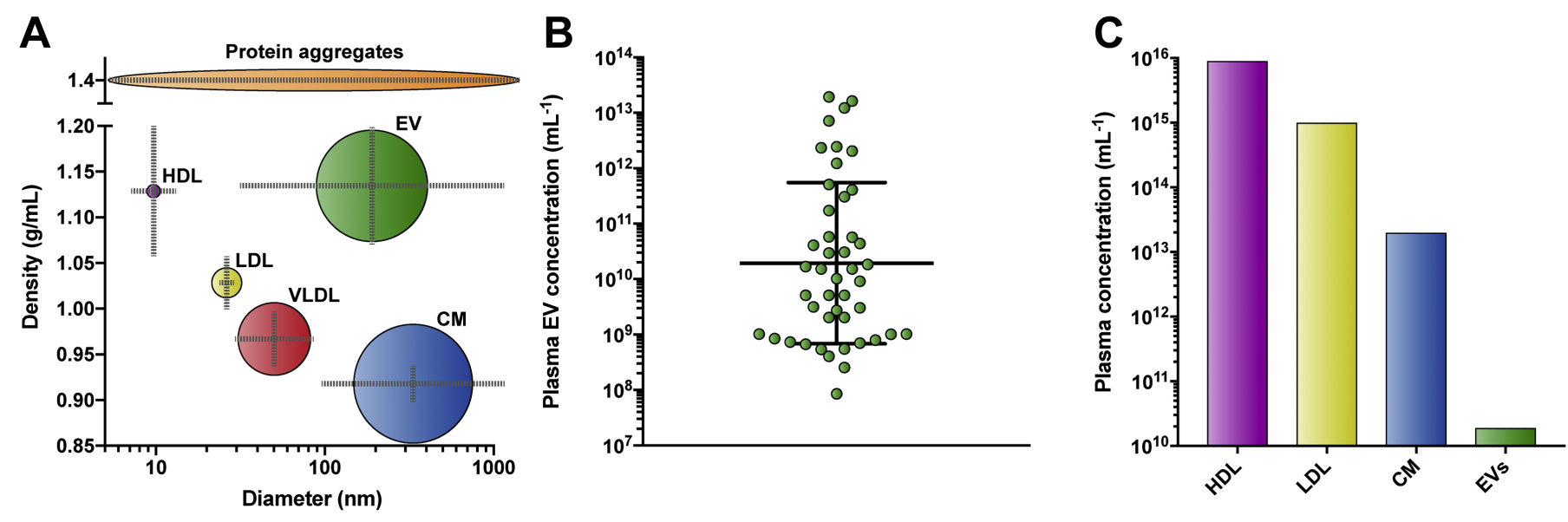

Figure 4 